Supplement of Hydrol. Earth Syst. Sci., 23, 1245-1261, 2019

https://doi.org/10.5194/hess-23-1245-2019-supplement

(c) Author(s) 2019. This work is distributed under

the Creative Commons Attribution 4.0 License.

(c) (1)

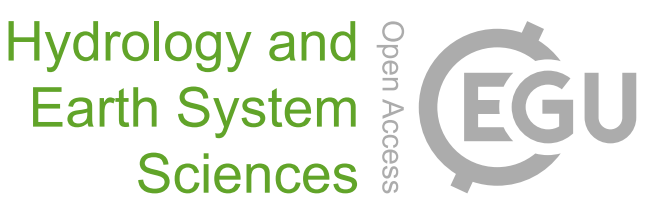

Supplement of

\title{
Multimodel assessments of human and climate impacts on mean annual streamflow in China
}

\section{Xingcai Liu et al.}

Correspondence to: Qiuhong Tang (tangqh@igsnrr.ac.cn)

The copyright of individual parts of the supplement might differ from the CC BY 4.0 License. 
Table S1. Main characteristics of human impacts in the GHMs used in this study.

\begin{tabular}{|c|c|c|c|}
\hline Model & Water use & Dam and Reservoirs & $\begin{array}{l}\text { Source of irrigation water } \\
\text { withdrawal }\end{array}$ \\
\hline $\mathrm{DBH}$ & modeled irrigation & $\begin{array}{l}\text { Use GRanD dataset, the number of dams and reservoirs varies } \\
\text { according to the construction year for the VARSOC runs. }\end{array}$ & river, reservoirs \\
\hline H08 & $\begin{array}{l}\text { modeled irrigation } \\
\text { prescribed domestic and } \\
\text { industrial water use }\end{array}$ & $\begin{array}{l}\text { Use GRanD dataset, the number of dams and reservoirs varies } \\
\text { according to the construction year for the VARSOC runs. }\end{array}$ & river, reservoirs, groundwater \\
\hline LPJmL & $\begin{array}{l}\text { modeled irrigation } \\
\text { prescribed domestic, industrial } \\
\text { and livestock }\end{array}$ & $\begin{array}{l}\text { Use GRanD dataset, the number of dams and reservoirs varies } \\
\text { according to the construction year for the VARSOC runs. } \\
\text { Evaporation from reservoir surface is calculated. }\end{array}$ & river, reservoirs \\
\hline MATSIRO & $\begin{array}{l}\text { modeled irrigation } \\
\text { prescribed domestic } \\
\text { industrial water use }\end{array}$ & $\begin{array}{l}\text { Use GRanD dataset, the number of dams and reservoirs varies } \\
\text { according to the construction year for the VARSOC runs. }\end{array}$ & river, reservoirs, groundwater \\
\hline PCR-GLOBWB & $\begin{array}{l}\text { modeled irrigation, domestic, } \\
\text { industrial and livestock water use }\end{array}$ & $\begin{array}{l}\text { Use GRanD dataset, the number of dams and reservoirs varies } \\
\text { according to the construction year for the VARSOC runs. } \\
\text { Evaporation from reservoir surface is calculated. }\end{array}$ & river, reservoirs, groundwater \\
\hline WaterGAP2 & $\begin{array}{l}\text { modeled irrigation, domestic, } \\
\text { industrial and livestock water use }\end{array}$ & $\begin{array}{l}\text { Use GRanD dataset, the number of dams and reservoirs varies } \\
\text { according to the construction year for the VARSOC runs. } \\
\text { Evaporation from reservoir surface is calculated. }\end{array}$ & $\begin{array}{l}\text { river, reservoirs, } \\
\text { groundwater }\end{array}$ \\
\hline
\end{tabular}


Table S2. The Nash-Sutcliffe coefficients (NSE) for the simulated monthly streamflow from VARSOC experiment and observed monthly streamflow $\left(\mathrm{m}^{3} \mathrm{~s}^{-1}\right.$ ) at the 44 stations over the 1971-2000 period. The observed mean annual streamflow (MAF, $\mathrm{m}^{3} \mathrm{~s}^{-1}$ ) averaged over the period is also shown for each station.

\begin{tabular}{|c|c|c|c|c|c|c|c|c|c|}
\hline Number & Station Name & MAF & NSE & River name & Number & Station Name & MAF & NSE & River name \\
\hline 1 & Guchengzi & 151.26 & -0.27 & Songhua River & 23 & Xixian & 117.87 & 0.31 & Huai River \\
\hline 2 & Fuyu & 449.68 & 0.53 & Songhua River & 24 & Fuyang & 117.74 & 0.63 & Huai River \\
\hline 3 & Tonghe & 1444.43 & 0.81 & Songhua River & 25 & Lutaizi & 639.00 & 0.80 & Huai River \\
\hline 4 & Kuerbin & 26.94 & 0.004 & Songhua River & 26 & Bengbu & 800.63 & 0.81 & Huai River \\
\hline 5 & Chaoyang & 18.00 & -0.88 & Liao River & 27 & Shishang & 1968.28 & 0.93 & Yangtze River \\
\hline 6 & Chifeng & 7.76 & -0.25 & Liao River & 28 & Changyang & 431.02 & 0.75 & Yangtze River \\
\hline 7 & Tieling & 84.36 & $<-1.0$ & Liao River & 29 & Pingshan & 4546.38 & 0.77 & Yangtze River \\
\hline 8 & Liaozhong & 101.43 & 0.50 & Liao River & 30 & Sinan & 910.98 & 0.79 & Yangtze River \\
\hline 9 & Changmapu & 29.30 & -0.37 & Northwest Rivers & 31 & Cuntan & 10747.92 & 0.68 & Yangtze River \\
\hline 10 & Yingluoxia & 51.06 & -0.26 & Northwest Rivers & 32 & Datong & 28460.19 & 0.78 & Yangtze River \\
\hline 11 & Zhamashenke & 22.70 & 0.09 & Northwest Rivers & 33 & Quzhou & 207.66 & 0.80 & Southeast Rivers \\
\hline 12 & Sandaohezi & 16.45 & $<-1.0$ & Hai River & 34 & Zhuji & 40.24 & 0.58 & Southeast Rivers \\
\hline 13 & Panjiakou & 60.87 & 0.01 & Hai River & 35 & Zhuqi & 1721.14 & 0.91 & Southeast Rivers \\
\hline 14 & Luanxian & 96.09 & 0.71 & Hai River & 36 & Yangkou & 442.85 & 0.72 & Southeast Rivers \\
\hline 15 & Xiapu & 4.58 & $<-1.0$ & Hai River & 37 & Daojieba & 1746.97 & 0.12 & Southwest Rivers \\
\hline 16 & Huangbizhuang & 32.14 & -0.07 & Hai River & 38 & Gulaohe & 96.63 & 0.22 & Southwest Rivers \\
\hline 17 & Cetian & 4.78 & -0.01 & Hai River & 39 & Manhao & 310.84 & 0.82 & Southwest Rivers \\
\hline 18 & Lanzhou & 976.80 & 0.53 & Yellow River & 40 & Jiangbianjie & 194.96 & 0.68 & Pearl River \\
\hline 19 & Shizuishan & 867.25 & 0.45 & Yellow River & 41 & Duanzhan & 2005.11 & 0.88 & Pearl River \\
\hline 20 & Longmen & 803.67 & -0.47 & Yellow River & 42 & Xiayan & 449.63 & 0.82 & Pearl River \\
\hline 21 & Huayuankou & 1103.51 & 0.09 & Yellow River & 43 & Wuxuan & 4130.25 & 0.81 & Pearl River \\
\hline 22 & Xianyang & 107.26 & 0.63 & Yellow River & 44 & Boluo & 782.04 & 0.80 & Pearl River \\
\hline
\end{tabular}


Table S3. The proportions of river segments of China categorized by MAF changes. " $<-10$ " in the header means the river segment showing MAF changes in $[-20 \%,-10 \%)$, and so on.

\begin{tabular}{|l|c|c|c|c|l|l|l|l|l|c|}
\hline & $<-\mathbf{3 0}$ & $<-\mathbf{2 0}$ & $\mathbf{- 1 0}$ & $<-5$ & $<\mathbf{0}$ & $<\mathbf{5}$ & $<\mathbf{1 0}$ & $<\mathbf{2 0}$ & $<\mathbf{3 0}$ & $>=\mathbf{3 0}$ \\
\hline$\Delta Q_{c}$ & 2.56 & 5.24 & 16.32 & 13.47 & 16.42 & 15.76 & 10.55 & 6.84 & 3.55 & 9.29 \\
\hline$\Delta Q_{h}$ & 2.28 & 1.00 & 3.83 & 9.04 & 54.27 & 26.80 & 1.29 & 0.85 & 0.13 & 0.50 \\
\hline$\Delta Q_{a}$ & 3.13 & 7.13 & 17.11 & 13.40 & 16.29 & 15.69 & 9.86 & 5.87 & 3.83 & 7.69 \\
\hline
\end{tabular}


Table S4. Ensemble members of streamflow changes ( $\Delta Q_{a}, \%$ of MAF) between 1971-1990 and 1991-2010.

\begin{tabular}{|c|c|c|c|c|c|c|c|c|c|c|c|c|}
\hline Forcing & Model & $\mathrm{CN}$ & SH & $\mathbf{L R}$ & NW & HA & YR & HU & $\mathbf{Y Z}$ & SE & SW & PR \\
\hline \multirow{6}{*}{ PGMFD v.2 } & H08 & -1.21 & -6.64 & -0.85 & 19.89 & -4.34 & -2.29 & 1.71 & -1.56 & -4.67 & 2.35 & 1.96 \\
\hline & $\mathrm{DBH}$ & 1.30 & -4.29 & -6.88 & 6.98 & -13.29 & -5.81 & 2.92 & 2.13 & -1.48 & 5.77 & 3.10 \\
\hline & LPJmL & 0.54 & -3.06 & -1.51 & 5.69 & -9.79 & -8.85 & 0.28 & 0.78 & -3.22 & 2.68 & 3.81 \\
\hline & PCR-GLOBWB & -1.04 & -7.51 & -5.99 & 6.00 & -7.80 & -2.32 & -5.50 & -0.95 & -2.95 & 3.13 & 0.79 \\
\hline & WaterGAP2 & 0.39 & -3.16 & -2.48 & 4.21 & -11.09 & -3.76 & 1.84 & 0.34 & -4.30 & 3.85 & 1.81 \\
\hline & MATSIRO & -2.43 & -10.04 & -3.90 & 31.15 & -6.60 & -6.37 & -0.51 & -2.67 & -4.94 & 1.77 & -1.44 \\
\hline \multirow{6}{*}{ GSWP3 } & H08 & -0.42 & -5.26 & -8.20 & 6.79 & -14.20 & -12.15 & 9.84 & 0.22 & 5.10 & -0.23 & 3.57 \\
\hline & $\mathrm{DBH}$ & -0.66 & -4.80 & -12.31 & 16.98 & -23.13 & -10.53 & 3.09 & 0.47 & 0.99 & 2.30 & -0.17 \\
\hline & LPJmL & -0.87 & -2.67 & -8.30 & 12.96 & -13.56 & -16.26 & 4.17 & -0.21 & 2.52 & -0.19 & 2.59 \\
\hline & PCR-GLOBWB & -2.14 & -6.90 & -8.17 & 11.08 & -12.07 & -7.35 & -0.84 & -1.43 & 1.37 & 0.60 & -1.12 \\
\hline & WaterGAP2 & -1.00 & -3.34 & -9.60 & 15.45 & -21.38 & -18.03 & 4.49 & -0.46 & 1.49 & 0.28 & 0.31 \\
\hline & MATSIRO & 1.09 & 0.73 & -11.25 & 22.81 & -33.18 & -24.49 & 6.21 & 1.72 & 2.79 & -1.13 & 2.37 \\
\hline \multirow{6}{*}{ WFDEI } & $\mathrm{H} 08$ & -6.74 & -7.50 & -6.16 & 4.51 & -18.85 & -14.18 & 3.31 & -6.50 & -4.76 & -4.34 & -6.75 \\
\hline & $\mathrm{DBH}$ & -6.38 & -8.75 & -11.96 & -3.84 & -26.26 & -18.47 & -4.47 & -5.05 & -3.46 & -2.85 & -6.48 \\
\hline & LPJmL & -4.12 & -4.16 & -7.66 & 8.27 & -14.78 & -16.88 & 0.61 & -3.29 & -4.59 & -3.73 & -4.84 \\
\hline & PCR-GLOBWB & 2.82 & -1.94 & -1.93 & 31.61 & -7.33 & -0.83 & -0.05 & 3.35 & 1.28 & 4.87 & 2.65 \\
\hline & WaterGAP2 & -4.69 & -4.77 & -8.08 & 9.55 & -22.00 & -23.05 & 0.62 & -3.88 & -5.04 & -3.87 & -5.27 \\
\hline & MATSIRO & 10.43 & 43.02 & 9.82 & 178.78 & -0.42 & 27.95 & 11.69 & 11.24 & 2.80 & 5.33 & 4.04 \\
\hline \multirow{3}{*}{ All ensembles } & Median & -0.93 & -4.53 & -7.27 & 10.32 & -13.43 & -9.69 & 1.78 & -0.34 & -2.22 & 1.19 & 1.30 \\
\hline & $25^{\text {th }}$ & -2.36 & -6.83 & -8.28 & 6.20 & -20.75 & -16.72 & 0.03 & -2.40 & -4.52 & -0.90 & -1.36 \\
\hline & $75^{\text {th }}$ & 0.51 & -3.09 & -2.84 & 19.16 & -8.30 & -4.27 & 3.95 & 0.70 & 1.46 & 3.01 & 2.64 \\
\hline
\end{tabular}


Table S5. Ensemble members of streamflow changes induced by climate variability ( $\Delta Q_{c}, \%$ of MAF) between $1971-1990$ and $1991-2010$.

\begin{tabular}{|c|c|c|c|c|c|c|c|c|c|c|c|c|}
\hline Forcing & Model & $\mathrm{CN}$ & SH & $\mathbf{L R}$ & $\mathbf{N W}$ & HA & YR & HU & $\mathbf{Y Z}$ & SE & SW & PR \\
\hline \multirow{6}{*}{ PGMFD v. 2} & H08 & -0.82 & -6.55 & -2.25 & 7.20 & -7.06 & -0.54 & 2.47 & -0.99 & -5.03 & 0.35 & 2.30 \\
\hline & $\mathrm{DBH}$ & 2.31 & -2.15 & -2.55 & 11.29 & -0.32 & 0.93 & 7.26 & 2.47 & -0.96 & 3.76 & 3.05 \\
\hline & LPJmL & 1.70 & -1.99 & 0.33 & 4.41 & -3.76 & 0.20 & 4.88 & 1.94 & -2.87 & 0.12 & 4.43 \\
\hline & PCR-GLOBWB & 0.33 & -5.87 & -3.44 & 1.09 & -2.98 & 0.21 & 2.90 & 0.42 & -2.24 & 1.34 & 2.23 \\
\hline & WaterGAP2 & 1.19 & -2.73 & -0.06 & 5.34 & -3.93 & -0.23 & 7.57 & 1.12 & -3.86 & 2.04 & 2.47 \\
\hline & MATSIRO & -3.79 & -13.87 & -8.12 & -5.06 & -14.63 & -20.25 & -8.49 & -3.69 & -4.73 & -2.05 & -1.90 \\
\hline \multirow{6}{*}{ GSWP3 } & H08 & 0.02 & -4.41 & -8.83 & 10.51 & -15.67 & -10.08 & 11.16 & 0.72 & 4.85 & -1.53 & 3.92 \\
\hline & $\mathrm{DBH}$ & 0.63 & -1.54 & -1.94 & 28.33 & -7.97 & -5.12 & 8.66 & 0.93 & 1.42 & 2.02 & -0.11 \\
\hline & LPJmL & 0.39 & -1.19 & -4.20 & 16.21 & -8.16 & -8.63 & 9.36 & 0.75 & 2.83 & -1.54 & 3.11 \\
\hline & PCR-GLOBWB & -0.72 & -5.45 & -5.20 & 10.00 & -8.34 & -5.57 & 6.40 & -0.16 & 1.93 & -0.05 & 0.21 \\
\hline & WaterGAP2 & -0.08 & -2.55 & -6.78 & 27.65 & -14.24 & -15.34 & 11.99 & 0.39 & 1.94 & -0.54 & 0.86 \\
\hline & MATSIRO & -0.77 & -3.21 & -16.35 & 12.44 & -53.34 & -40.28 & 5.67 & 0.19 & 3.03 & -3.49 & 1.70 \\
\hline \multirow{6}{*}{ WFDEI } & H08 & -6.12 & -7.13 & -6.60 & 5.11 & -19.75 & -11.21 & 4.52 & -5.75 & -5.03 & -5.93 & -6.47 \\
\hline & $\mathrm{DBH}$ & -4.87 & -6.37 & -6.11 & 2.70 & -13.98 & -12.34 & 1.91 & -4.00 & -2.90 & -4.28 & -6.02 \\
\hline & LPJmL & -2.73 & -2.91 & -4.42 & 12.00 & -10.50 & -9.73 & 6.45 & -1.97 & -4.10 & -4.83 & -4.04 \\
\hline & PCR-GLOBWB & 4.71 & -0.30 & 1.13 & 30.71 & -2.89 & 1.44 & 7.22 & 4.99 & 1.97 & 4.93 & 4.05 \\
\hline & WaterGAP2 & -3.77 & -4.03 & -5.46 & 17.37 & -15.51 & -18.55 & 8.14 & -2.93 & -4.44 & -5.38 & -4.47 \\
\hline & MATSIRO & -1.28 & 1.25 & -6.82 & 96.98 & -13.82 & -20.52 & 4.85 & -0.40 & -0.52 & -3.67 & -3.94 \\
\hline \multirow{3}{*}{ All ensembles } & Median & -0.29 & -3.06 & -4.81 & 17.86 & -9.42 & -9.35 & 6.43 & 0.17 & -1.60 & 0.55 & 1.28 \\
\hline & $25^{\text {th }}$ & -2.24 & -5.77 & -6.73 & 11.71 & -14.53 & -14.85 & 4.61 & -1.89 & -4.04 & -1.17 & -3.43 \\
\hline & $75^{\text {th }}$ & 0.49 & -2.03 & -2.32 & 33.21 & -4.71 & -0.51 & 7.99 & 0.76 & 1.94 & 2.82 & 2.90 \\
\hline
\end{tabular}


Table S6. Ensemble members of streamflow changes induced by DHI change ( $\Delta Q_{h}, \%$ of MAF) between 1971-1990 and $1991-2010$.

\begin{tabular}{|c|c|c|c|c|c|c|c|c|c|c|c|c|}
\hline Forcing & Model & $\mathrm{CN}$ & SH & LR & NW & HA & YR & HU & $\mathbf{Y Z}$ & SE & SW & PR \\
\hline \multirow{6}{*}{ PGMFD v.2 } & H08 & -0.42 & -0.09 & 1.39 & -2.93 & 2.72 & -1.51 & -0.76 & -0.44 & 0.36 & -0.06 & -0.34 \\
\hline & $\mathrm{DBH}$ & -0.98 & -2.13 & -4.33 & -6.68 & -12.98 & -6.49 & -4.35 & -0.20 & -0.52 & -0.08 & 0.06 \\
\hline & LPJmL & -1.40 & -1.07 & -1.85 & -2.53 & -6.03 & -8.76 & -4.60 & -1.11 & -0.35 & -0.27 & -0.62 \\
\hline & PCR-GLOBWB & -1.32 & -1.64 & -2.55 & -0.93 & -4.83 & -2.24 & -8.39 & -1.12 & -0.71 & -0.19 & -1.44 \\
\hline & WaterGAP2 & -0.86 & -0.43 & -2.42 & -4.40 & -7.15 & -3.28 & -5.73 & -0.67 & -0.44 & -0.05 & -0.66 \\
\hline & MATSIRO & 1.09 & 3.83 & 4.22 & -4.91 & 8.03 & 14.39 & 7.98 & 1.10 & -0.21 & 0.01 & 0.45 \\
\hline \multirow{6}{*}{ GSWP3 } & H08 & -0.51 & -0.85 & 0.63 & -5.07 & 1.47 & -1.88 & -1.33 & -0.44 & 0.25 & -0.05 & -0.35 \\
\hline & $\mathrm{DBH}$ & -1.12 & -3.26 & -10.37 & -12.44 & -15.16 & -5.24 & -5.57 & -0.31 & -0.43 & -0.08 & -0.06 \\
\hline & LPJmL & -1.31 & -1.48 & -4.10 & -4.66 & -5.40 & -7.31 & -5.19 & -0.87 & -0.31 & -0.25 & -0.52 \\
\hline & PCR-GLOBWB & -1.14 & -1.45 & -2.97 & -1.63 & -3.72 & -1.59 & -7.24 & -0.95 & -0.56 & -0.15 & -1.33 \\
\hline & WaterGAP2 & -0.93 & -0.79 & -2.82 & -12.67 & -7.13 & -2.33 & -7.50 & -0.79 & -0.45 & -0.03 & -0.54 \\
\hline & MATSIRO & 1.59 & 3.94 & 5.10 & -6.67 & 20.16 & 16.41 & 0.54 & 1.57 & -0.25 & 0.17 & 0.67 \\
\hline \multirow{6}{*}{ WFDEI } & H08 & -0.65 & -0.37 & 0.44 & -4.36 & 0.89 & -2.76 & -1.21 & -0.58 & 0.26 & -0.05 & -0.28 \\
\hline & $\mathrm{DBH}$ & -1.45 & -2.38 & -5.85 & -5.73 & -12.28 & -5.93 & -6.38 & -0.87 & -0.56 & -0.05 & -0.46 \\
\hline & LPJmL & -1.43 & -1.25 & -3.24 & -6.81 & -4.28 & -6.81 & -5.84 & -1.13 & -0.49 & -0.30 & -0.80 \\
\hline & PCR-GLOBWB & -1.32 & -1.63 & -3.06 & -3.35 & -4.44 & -1.98 & -7.27 & -1.07 & -0.69 & -0.16 & -1.40 \\
\hline & WaterGAP2 & -1.03 & -0.73 & -2.62 & -12.11 & -6.48 & -4.21 & -7.52 & -0.86 & -0.60 & -0.02 & -0.79 \\
\hline & MATSIRO & 11.57 & 41.77 & 16.64 & -5.89 & 13.41 & 49.06 & 6.84 & 11.98 & 3.32 & 7.34 & 7.98 \\
\hline \multirow{3}{*}{ All ensembles } & Median & -1.04 & -0.96 & -2.58 & -7.96 & -4.63 & -2.60 & -5.38 & -0.74 & -0.44 & -0.07 & -0.49 \\
\hline & $25^{\text {th }}$ & -1.40 & -1.60 & -3.20 & -15.58 & -6.97 & -5.84 & -7.03 & -0.96 & -0.55 & -0.19 & -0.76 \\
\hline & $75^{\text {th }}$ & -0.57 & -0.39 & 0.58 & -5.90 & 1.33 & -1.71 & -1.24 & -0.35 & -0.22 & -0.05 & -0.11 \\
\hline
\end{tabular}


Table S7. Ensemble medians, $25^{\text {th }}$ and $75^{\text {th }}$ percentiles of MAF changes (\%) induced by DHI change $\left(\Delta Q_{h}\right)$ from 1971-1980 to 1981-1990, 1991-2000, and 2001-2010, respectively. All $\Delta Q_{h}$ values are percentages of the MAF from VARSOC simulations over the 1971-1980 period.

\begin{tabular}{|c|c|c|c|c|c|c|c|c|c|}
\hline \multirow{2}{*}{ Region } & \multicolumn{3}{|c|}{$\mathbf{1 9 8 1 - 1 9 9 0}$} & \multicolumn{3}{|c|}{$\mathbf{1 9 9 1 - 2 0 0 0}$} & \multicolumn{3}{c|}{ 2001-2010 } \\
\cline { 2 - 10 } & $\Delta Q_{h}$ & $\Delta Q_{h} 25$ th & $\Delta Q_{h_{-}} 75$ th & $\Delta Q_{h}$ & $\Delta Q_{h_{-}} 25$ th & $\Delta Q_{h_{-}} 75$ th & $\Delta Q_{h}$ & $\Delta Q_{h_{-}} 25$ th & $\Delta Q_{h_{-} 75 \text { th }}$ \\
\hline CN & -0.37 & -0.58 & 0.05 & -0.65 & -1.39 & 0.89 & -1.62 & -1.94 & -0.78 \\
\hline SHJ & -1.78 & -2.02 & -0.34 & -1.46 & -2.01 & -0.97 & -2.15 & -2.57 & -1.47 \\
\hline LR & -3.43 & -4.38 & -0.23 & -2.30 & -3.18 & 1.92 & -5.21 & -8.29 & -3.67 \\
\hline NW & -6.09 & -8.15 & -4.18 & -8.99 & -13.12 & -4.58 & -13.53 & -25.40 & -9.97 \\
\hline HA & -2.81 & -7.40 & -0.98 & -4.49 & -6.58 & 2.09 & -7.07 & -10.65 & 1.89 \\
\hline YR & -7.49 & -13.76 & -3.83 & -4.10 & -6.46 & -1.83 & -8.95 & -10.95 & -3.71 \\
\hline HU & -1.97 & -3.74 & -0.03 & -5.53 & -7.41 & -2.20 & -7.01 & -10.35 & -0.54 \\
\hline YZ & 0.05 & -0.19 & 0.64 & -0.38 & -0.93 & 1.39 & -0.73 & -1.18 & 0.37 \\
\hline SE & -0.32 & -0.42 & -0.16 & -0.37 & -0.58 & -0.18 & -0.85 & -1.01 & -0.46 \\
\hline SW & -0.03 & -0.06 & -0.01 & -0.07 & -0.19 & -0.04 & -0.08 & -0.30 & -0.06 \\
\hline PR & -0.31 & -0.54 & -0.14 & -0.75 & -1.32 & -0.22 & -0.42 & -0.78 & -0.19 \\
\hline
\end{tabular}


Table S8. Relative contributions of DHI from previous studies. $\Delta Q_{a}$ denotes the relative contribution of DHI and is computed as $100 \times \Delta Q_{h} / \Delta Q_{a}$ in the studies. Period 1 denotes the period without (or with little) human impact, Period 2 denotes the period with human impact. Period 2 is blank when no sub-periods were used in the study.

\begin{tabular}{|c|c|c|c|c|c|c|c|c|c|}
\hline Major River & River & $\Delta Q_{a}(\%)$ & Period 1 & Period 2 & Station & Latitude & Longitude & $\begin{array}{l}\text { Catchment } \\
\text { area }\left(\mathbf{k m}^{2}\right)\end{array}$ & Reference \\
\hline \multirow[t]{3}{*}{ Hai River } & Qinlong River & -41.5 & $1957-1979$ & 1980-2000 & Taolinkou & 40.13 & 119.05 & 5060 & \multirow{2}{*}{ Bao et al., 2012} \\
\hline & Zhang River & -73.9 & 1951-1972 & $1973-2004$ & Guantai & 36.33 & 114.08 & 17800 & \\
\hline & Chao River & -68.6 & $\begin{array}{c}1961-1966, \\
1973-1979\end{array}$ & $1980-2001$ & & 41.00 & 117.00 & 6716 & Wang et al., 2009 \\
\hline \multirow[t]{5}{*}{ Yellow River } & Upper reaches & -37 & 1956-1989 & $1990-2000$ & Tangnaihai & 35.50 & 100.15 & 121972 & \multirow{2}{*}{ Zhao et al., 2009} \\
\hline & Upper reaches & -46 & 1968-1986 & $1987-2000$ & Lanzhou & 36.07 & 103.82 & 222551 & \\
\hline & Upper reaches & -44 & 1960-1970 & $1991-2000$ & Baimasi & 34.72 & 112.58 & 13915 & Wang et al., 2010 \\
\hline & Wuding River & -84.3 & 1961-1971 & 1972-1997 & Baijiachuan & 37.24 & 110.42 & 30261 & Li et al., 2007 \\
\hline & Wuding River & -23 & 1961-2005 & & Baijiachuan & 37.24 & 110.42 & 30261 & Yuan et al. 2018 \\
\hline
\end{tabular}



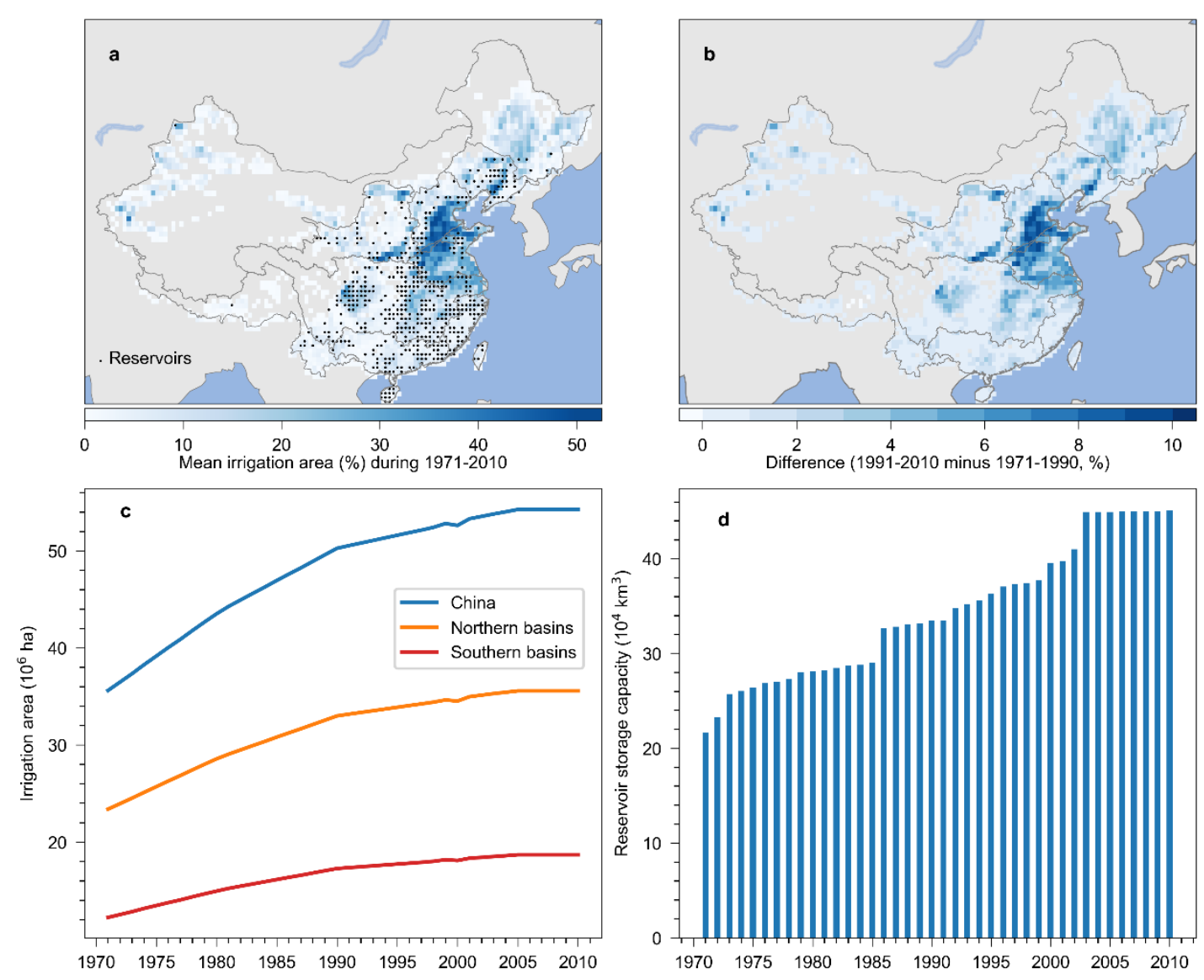

Figure S1. Irrigated areas (Fader et al., 2010; Portmann et al., 2010) and reservoirs (Lehner et al., 2011) in China used in the ISIMIP2a VARSOC experiment. (a): mean irrigation area per grid cell (\%) over the 1971-2010 period and locations of reservoir; (b): difference in mean irrigation area between the periods of 1971-1990 and 1991 and 2010; (c): annual irrigation area for China, northern basins, and southern basins; (d): annual storage capacity of reservoirs in China. The areas without irrigation are not shown on the map. 

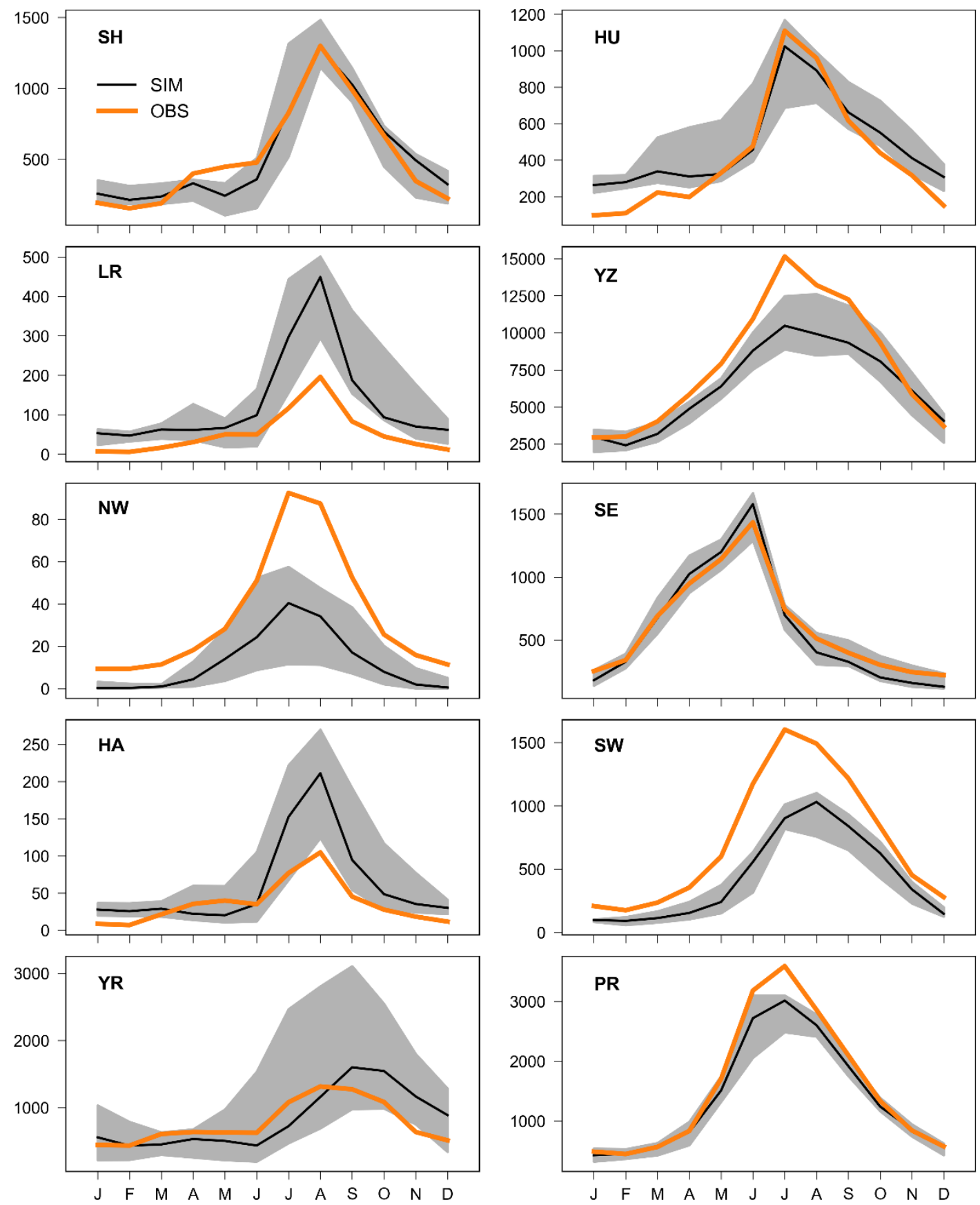

Figure S2. The seasonal cycle of streamflow from observations and GHMs. The seasonal observations are based on monthly streamflow and averaged for the hydrological stations in each basin (Figure 1). The simulations are averaged values over the grid cells identified by the location of stations. SIM indicates simulations and OBS indicates observations. The grey areas show the 25th and 75th percentiles of the multimodel simulations. Northern basins: Songhua River (SH), Liao River (LR), Northwest Rivers (NW), Hai River (HA), Yellow River (YR), Huai River (HU); Southern basins: Yangtze River (YZ), Southeast Rivers (SE), Southwest Rivers (SW), Pearl River (PR). 

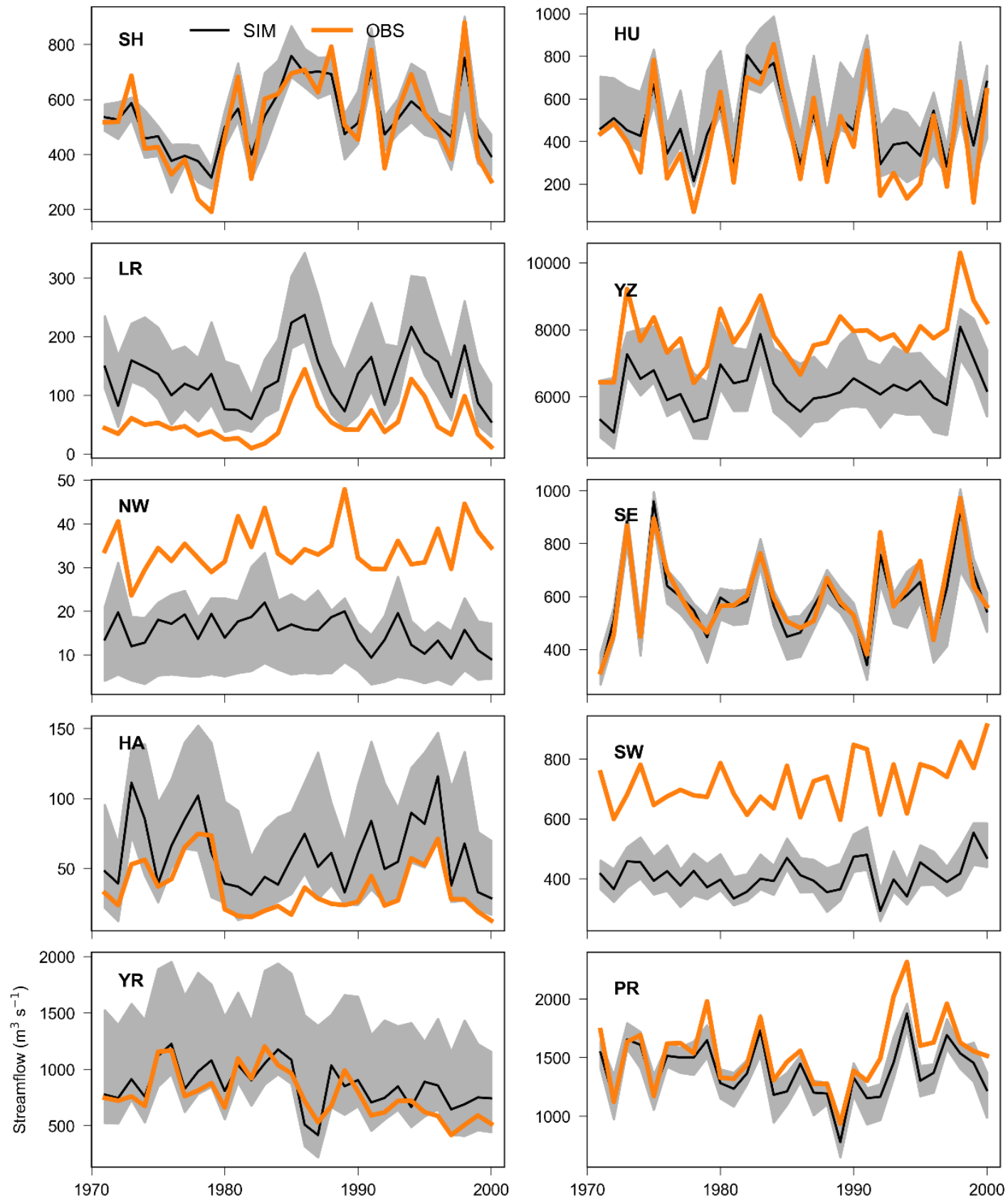

Figure S3. Simulated (black) and observed (orange) mean annual streamflow at the hydrological stations in each basin. The observations are the average values of the hydrological stations, while the simulations are averaged values over the grid cells identified by the location of stations. The grey areas show the 25th and 75th percentiles of the multimodel simulations. 

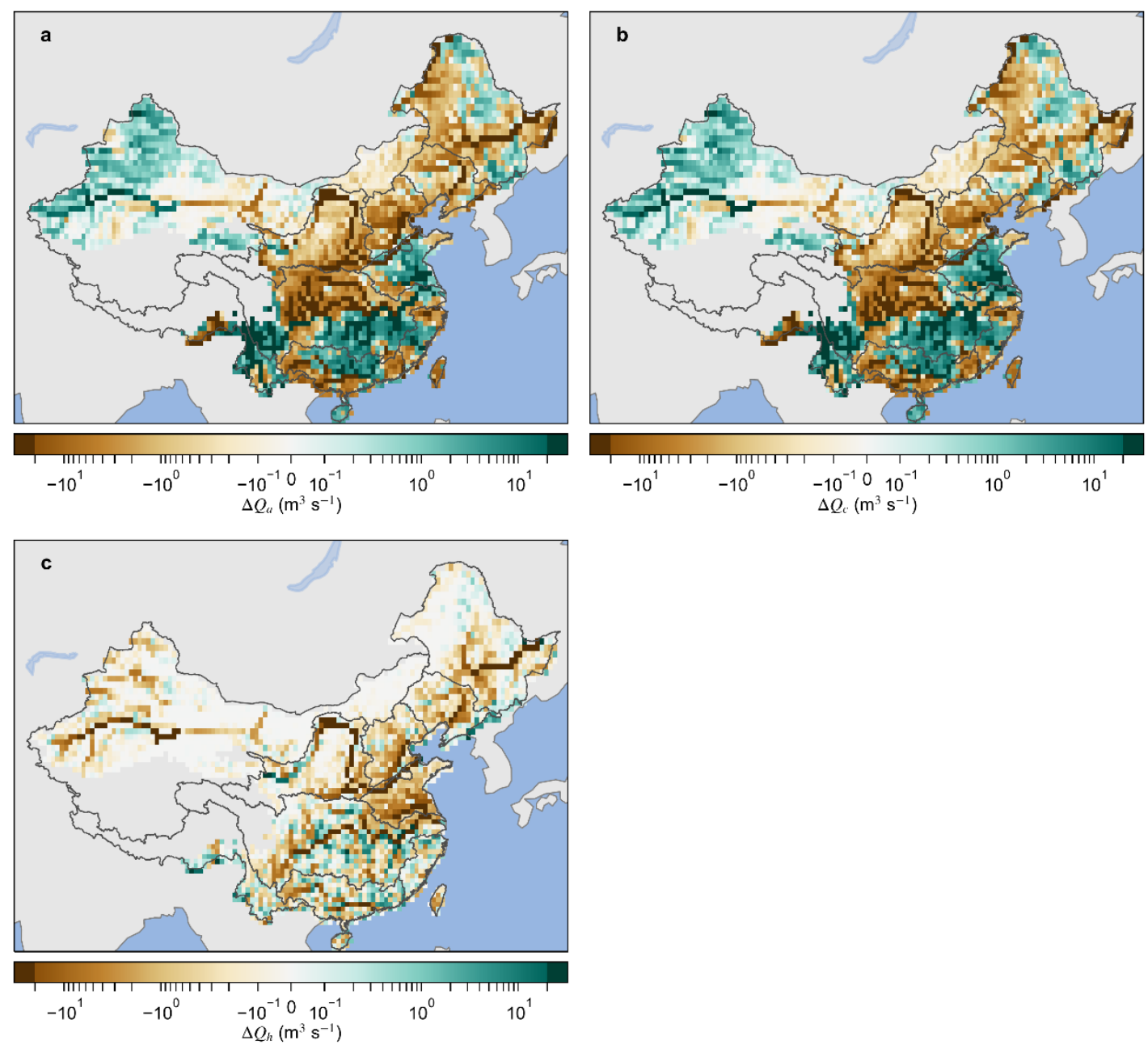

Figure S4. MAF changes $\left(\mathrm{m}^{3} \mathrm{~s}^{-1}\right)$ over China between the sub-periods 1971-1990 and 19912010. (a) Total MAF changes $\left(\Delta Q_{a}\right)$, (b) MAF changes induced by climate variability $\left(\Delta Q_{c}\right)$ and (c) MAF changes induced by DHI change $\left(\Delta Q_{h}\right)$. 

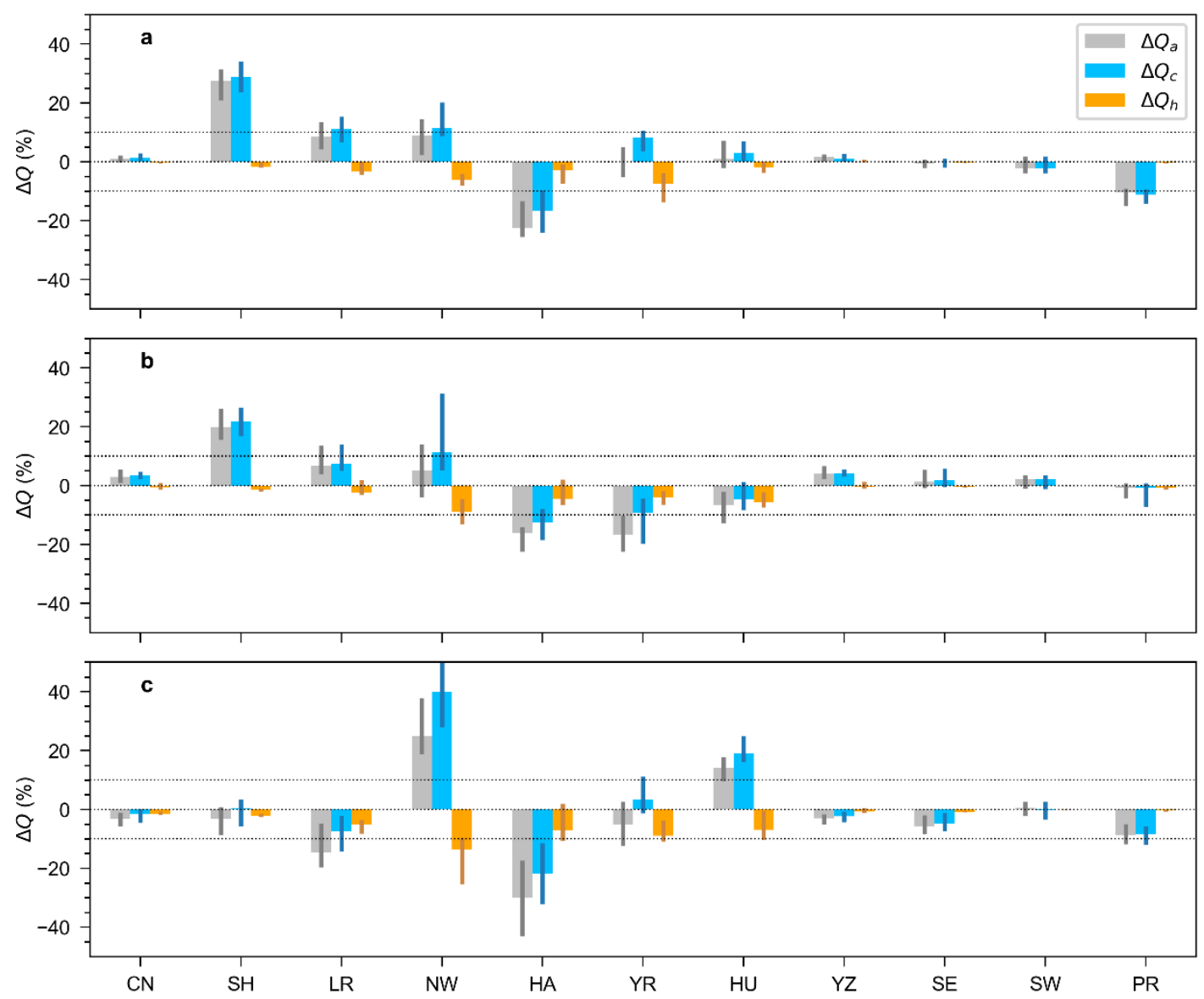

Figure $\mathrm{S} 5$. Total MAF change $\left(\Delta Q_{a}\right)$, MAF change induced by climate variability $\left(\Delta Q_{c}\right)$, and MAF change induced by DHI change $\left(\Delta Q_{h}\right)$ from the period 1971-1980 to (a) 1981-1990, (b) 1991-2000 and (c) 2001-2010, respectively. The bars show the medians and the error bars show the range of 25th and 75th of MAF changes. 


\section{Reference}

Bao, Z., Zhang, J., Wang, G., Fu, G., He, R., Yan, X., Jin, J., Liu, Y., and Zhang, A.: Attribution for decreasing streamflow of the Haihe River basin, northern China: Climate variability or human activities?, J. Hydrol., 460-461, 117-129, 10.1016/j.jhydrol.2012.06.054, 2012.

Fader, M., Rost, S., Müller, C., Bondeau, A., and Gerten, D.: Virtual water content of temperate cereals and maize: Present and potential future patterns, J. Hydrol., 384, 218-231, 10.1016/j.jhydrol.2009.12.011, 2010.

Lehner, B., Liermann, C. R., Revenga, C., Vörösmarty, C., Fekete, B., Crouzet, P., Döll, P., Endejan, M., Frenken, K., Magome, J., Nilsson, C., Robertson, J. C., Rödel, R., Sindorf, N., and Wisser, D.: High-resolution mapping of the world's reservoirs and dams for sustainable river-flow management, Frontiers in Ecology and the Environment, 9, 494-502, 10.1890/100125, 2011.

Li, L., Zhang, L., Wang, H., Wang, J., Yang, J., Jiang, D., Li, J., and Qin, D.: Assessing the impact of climate variability and human activities on streamflow from the Wuding River basin in China, Hydrol. Processes, 21, 3485-3491, doi:10.1002/hyp.6485, 2007.

Ma, F., Ye, A., Gong, W., Mao, Y., Miao, C., and Di, Z.: An estimate of human and natural contributions to flood changes of the Huai River, Global Planet. Change, 119, 39-50, 10.1016/j.gloplacha.2014.05.003, 2014.

Portmann, F. T., Siebert, S., and Döll, P.: MIRCA2000 — Global monthly irrigated and rainfed crop areas around the year 2000: A new high-resolution data set for agricultural and hydrological modeling, Global Biogeochem. Cycles, 24, GB1011, 10.1029/2008GB003435, 2010.

Wang, G., Xia, J., and Chen, J.: Quantification of effects of climate variations and human activities on runoff by a monthly water balance model: A case study of the Chaobai River basin in northern China, Water Resour. Res., 45, doi:10.1029/2007WR006768, 2009. Wang, J., Hong, Y., Gourley, J., Adhikari, P., Li, L., and Su, F.: Quantitative assessment of climate change and human impacts on long-term hydrologic response: a case study in a subbasin of the Yellow River, China, Int. J. Climatol., 30, 2130-2137, doi:10.1002/joc.2023, 2010.

Yuan, X., Jiao, Y., Yang, D., and Lei, H.: Reconciling the Attribution of Changes in Streamflow Extremes From a Hydroclimate Perspective, Water Resour. Res., doi:10.1029/2018WR022714, 2018.

Zhao, F., Xu, Z., Zhang, L., and Zuo, D.: Streamflow response to climate variability and human activities in the upper catchment of the Yellow River Basin, Sci. China Ser. E: Technol. Sci., 52, 3249, 10.1007/s11431-009-0354-3, 2009. 